\title{
Direct C-Glycosylation by Indium-Mediated Alkynylation on Anomeric Position
}

Nadège Lubin-Germain ${ }^{\dagger *}$, Jean-Pierre Baltaze ${ }^{ \pm}$, Alexis Coste ${ }^{\dagger}$, Agnès Hallonet ${ }^{\dagger}$, Hugo Lauréano ${ }^{\dagger}$, Grégory Legrave ${ }^{\dagger}$, Jacques Uziel ${ }^{\dagger *}$ and Jacques Augé ${ }^{\dagger}$

Laboratoire de Synthèse Organique Sélective et Chimie Organométallique Unité CNRS-UCP-ESCOM 8123, 5 mail GayLussac, Neuville-sur-Oise, 95031 Cergy-Pontoise, France and ICMMO - UMR 8182 Bâtiment 420 - Université Paris-Sud XI 15 rue Georges Clémenceau 91405 Orsay Cedex Francel.

Corresponding authors :

nadege.lubin-germain@u-cergy.fr

\footnotetext{
$1 \dagger$ University of Cergy-Pontoise.

${ }^{ \pm}$University of Paris-Sud.
} 


\section{Table of Contents}

Section A : general informations 3

$\begin{array}{ll}\text { Section B : experimental procedures } & 4\end{array}$

Section $\mathrm{C}:{ }^{1} \mathrm{H}$ and ${ }^{13} \mathrm{C}$ NMR spectra 5 


\section{Section A : general informations}

All reactions were conducted under an atmosphere of argon using standard Schlenk techniques. Metallic indium and indium salts were purchased from Aldrich. Dichloromethane was distilled under argon from $\mathrm{CaH}_{2} \cdot{ }^{1} \mathrm{H}$ and ${ }^{13} \mathrm{C}$ NMR spectra were recorded in $\mathrm{CDCl}_{3}$ on a Brucker DPX-250 and on a Jeol $400 \mathrm{Mz}$ ECX. High resolution mass spectra were obtained with an MS Jeol 700 spectrometer or a LTQ orbitrap spectrometer. Optical rotations were determined at $25^{\circ} \mathrm{C}$ in $\mathrm{CHCl}_{3}, 589 \mathrm{~nm}$, on a JASPO DIP 370 instrument and IR spectra were recorded on a Bruker Tensor 27 spectrophotometer. Melting points (uncorrected) were determined on a Buchi B-545. Elemental analyses were done at the Central Service of Analysis (CNRS, Vernaison). Thin layer chromatography was carried out on silica gel plates (Macherey-Nagel), spots were detected with UV light and revealed with $\mathrm{H}_{2} \mathrm{SO}_{4}$ solution. Flash chromatography was performed with silica gel 60, 40-63 $\mu \mathrm{m}$. 


\section{Section B : experimental procedures}

\section{Typical procedure for alkynylation reaction}

$2.4 \mathrm{mmol}$ of indium powder was placed in a Schlenk tube under vacuum/argon. In another hand, the protected carbohydrate was added at a solution of iodoacetylene $(2 \mathrm{mmol})$ in $6 \mathrm{~mL}$ of dichloromethane. This solution was then transferred on the indium. The suspension was stirred during $24 \mathrm{~h}$ under reflux and monitored by tlc (cyclohexaneAcOEt : 80/20). $20 \mathrm{~mL}$ of a $10 \%$ solution of $\mathrm{NaHCO}_{3}$ were added and then the mixture was extracted with $2 \times 20 \mathrm{~mL}$ of dichloromethane. The combined organic layers were dried $\left(\mathrm{MgSO}_{4}\right)$. After filtration, the solvent was removed under reduced pressure. The crude was purified by flash chromatography on silica gel.

\section{Typical procedure for alkyne iodination}

To a solution of diiodine $(1.09 \mathrm{mmol})$ in $2 \mathrm{~mL}$ of benzene under argon, morpholine $(305 \mu \mathrm{L})$ was slowly added. The reaction was stirred to obtain an orange heterogeneous solution.

A solution of alkyne $(1 \mathrm{mmol})$ in dichloromethane $(2 \mathrm{~mL})$ was added slowly and the medium was warmed to $50^{\circ} \mathrm{C}$. The reaction was monitored by tlc (cyclohexane / ethyl acetate 8/2). At completion, the solution was filtered on celite and washed with diethyl ether. The organic layer was successively washed with $\mathrm{NH}_{4} \mathrm{Cl}$ sat., $\mathrm{NaHCO}_{3}$ sat., $10 \%$ $\mathrm{Na}_{2} \mathrm{~S}_{2} \mathrm{O}_{3}$ and $\mathrm{NaCl}$ sat. After evaporation, the crude was purified by flash chromatography (cyclohexane / AcOEt 95/5) to afford the iodoalkyne.

\section{Synthesis of disaccharide 25}

To a solution of $C$-disaccharide 24 (124 mg, $0.170 \mathrm{mmol})$ in $5 \mathrm{~mL}$ of $\mathrm{MeOH} /$ ethyl acetate $(1 / 1), \mathrm{Pd} / \mathrm{C}(45 \mathrm{mg})$ was added. The medium was stirred under hydrogen pressure (40 psi) during $24 \mathrm{~h}$. When the reaction was completed, the suspension was filtered on celite, washed with methanol and concentrated under vacuum.

The crude was directly acetylated by a classical procedure $\left(5 \mathrm{~mL}\right.$ of $\mathrm{Ac}_{2} \mathrm{O}, 10 \mathrm{~mL}$ of pyridine and $22 \mathrm{mg}$ of DMAP). After $7 \mathrm{~h}$, the reaction was diluted in $20 \mathrm{~mL}$ of diethyl ether and the organic layer was washed with $\mathrm{NH}_{4} \mathrm{Cl}$ saturated solution, then $\mathrm{NaCl}$ saturated solution, and dried on $\mathrm{MgSO}_{4}$. After filtration and concentration under vacuum, the crude was purified by flash chromatography (cyclohexane / ethyl acetate 7/3) to afford the compound 25 as a colorless solid. 
Section $\mathrm{C}:{ }^{1} \mathrm{H}$ and ${ }^{13} \mathrm{C}$ NMR spectra

\section{Compound 2a}

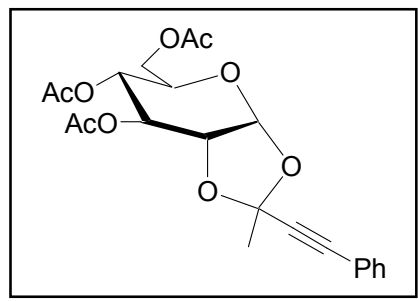

Obtained according to the typical procedure of alkynylation.

HRMS-CI : $m / z[\mathrm{M}+\mathrm{H}]^{+}$calcd for $\mathrm{C}_{22} \mathrm{H}_{25} \mathrm{O}_{9}: 433.1499$; found : 433.1494.

IR (ATR): 690, 755, 1221, 1741, $2234 \mathrm{~cm}^{-1}$.

${ }^{1} \mathrm{H}$ NMR $\left(400 \mathrm{MHz}, \mathrm{CDCl}_{3}\right): \delta 1.9\left(\mathrm{~s}, 3 \mathrm{H}, \mathrm{CH}_{3}\right), 2.1(2 \mathrm{~s}, 6 \mathrm{H}, \mathrm{Ac}), 4.02\left(\mathrm{ddd}, 1 \mathrm{H}, J=2.8,5.04,9.5 \mathrm{~Hz}, \mathrm{H}_{5}\right), 4.16(\mathrm{dd}$, $\left.1 \mathrm{H}, J=2.8,11.9 \mathrm{~Hz}, \mathrm{H}_{6}\right), 4.21\left(\mathrm{dd}, 1 \mathrm{H}, J=5.04,11.9 \mathrm{~Hz}, \mathrm{H}_{6}\right.$ ), 4.4 (ddd, $1 \mathrm{H}, J=0.9,2.3,5.04 \mathrm{~Hz}, \mathrm{H}_{2}$ ), 3.67 (ddd, $1 \mathrm{H}$, $\left.J=0.9,2.3,9.5 \mathrm{~Hz}, \mathrm{H}_{4}\right), 3.99\left(\mathrm{dd}, 1 \mathrm{H}, J=2.3 \mathrm{~Hz}, \mathrm{H}_{3}\right), 4.5\left(\mathrm{~d}, 1 \mathrm{H}, J=5.04 \mathrm{~Hz}, \mathrm{H}_{1}\right) .{ }^{13} \mathrm{C}$ NMR $\left(100 \mathrm{MHz}, \mathrm{CDCl}_{3}\right): \delta$ 20.7, 26.4, 62.9, 66.6, 68.2, 69.9, 73.5, 83.7, 86, 96.8, 101.1, 121.2, 128.2, 129.0, 131.7, 169.1, 169.6, 170.7.

\section{Compound 4}

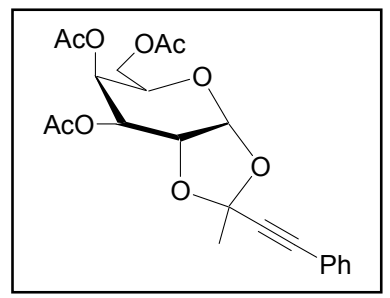

Obtained according to the typical procedure of alkynylation.

HRMS-CI : $m / z[\mathrm{M}+\mathrm{H}]^{+}$calcd for $\mathrm{C}_{22} \mathrm{H}_{25} \mathrm{O}_{9}: 433.1499$; found : 433.1497.

IR (ATR): 691, 758, 1217, 1746, $2236 \mathrm{~cm}^{-1}$.

4 major: ${ }^{1} \mathrm{H}$ NMR $\left(400 \mathrm{MHz}, \mathrm{CDCl}_{3}\right): \delta 1.8\left(\mathrm{~s}, 3 \mathrm{H}, \mathrm{CH}_{3}\right), 2.1(\mathrm{~m}, 9 \mathrm{H}, \mathrm{Ac}), 4.1\left(\mathrm{~m}, 2 \mathrm{H}, \mathrm{H}_{6}\right), 4.3(\mathrm{dd}, 1 \mathrm{H}, J=5.04,6.88$ $\left.\mathrm{Hz}, \mathrm{H}_{2}\right), 4.37$ (ddd, $\left.1 \mathrm{H}, J=1.84,6.44,8.28 \mathrm{~Hz}, \mathrm{H}_{5}\right), 5.06\left(\mathrm{dd}, 1 \mathrm{H}, J=3.6,6.8 \mathrm{~Hz}, \mathrm{H}_{3}\right), 5.41(\mathrm{dd}, 1 \mathrm{H}, J=1.84,3.6 \mathrm{~Hz}$, $\left.\mathrm{H}_{4}\right), 5.88\left(\mathrm{~d}, 1 \mathrm{H}, J=5.04 \mathrm{~Hz}, \mathrm{H}_{1}\right) 7.2-7.4(\mathrm{~m}, 5 \mathrm{H}, \mathrm{Ar}) .{ }^{13} \mathrm{C} \mathrm{NMR}\left(100 \mathrm{MHz}, \mathrm{CDCl}_{3}\right): \delta 20.7,61.6,65.8,69.2,71.6$, $71.9,83.8,86.8,121.4,128.2,128.9,131.7,169.9,170.1,169.9$.

4 minor: ${ }^{1} \mathrm{H}$ NMR $\left(400 \mathrm{MHz}, \mathrm{CDCl}_{3}\right): \delta 2.1(\mathrm{~m}, 9 \mathrm{H}, \mathrm{Ac}), 5(\mathrm{dd}, 1 \mathrm{H}, J=3.2,6.4 \mathrm{~Hz}, \mathrm{H} 3), 5.88(\mathrm{~d}, 1 \mathrm{H}, J=4.56 \mathrm{~Hz}$, H1), 7.2-7.4 (m, 5H).

\section{Compound 6}

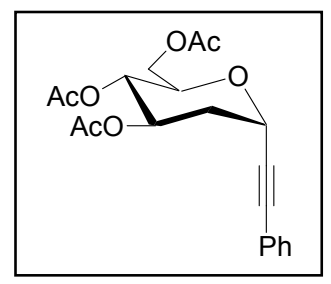

Obtained according to the typical procedure of alkynylation. 
HRMS-CI : $m / z[\mathrm{M}+\mathrm{H}]^{+}$calcd for $\mathrm{C}_{20} \mathrm{H}_{23} \mathrm{O}_{7}: 375.1444$; found 375.1440 .

IR (ATR): 700, 1224, $1735 \mathrm{~cm}^{-1}$.

${ }^{1} \mathrm{H}$ NMR (400MHz, $\left.\mathrm{CDCl}_{3}\right): \delta 2.1(\mathrm{~m}, 9 \mathrm{H}, \mathrm{Ac}), 2.27$ (ddd, $1 \mathrm{H}, J=12.8,5.04,1.36 \mathrm{~Hz}, \mathrm{H}_{2 \mathrm{eq}}$ ), 4.03 (dd, $1 \mathrm{H}, J=1.8$, $\left.12.4 \mathrm{~Hz}, \mathrm{H}_{6}\right), 4.16\left(\mathrm{ddd}, 1 \mathrm{H}, J=1.8,4.12,9.6 \mathrm{~Hz}, \mathrm{H}_{5}\right), 4.29\left(\mathrm{dd}, 1 \mathrm{H}, J=4.12,12.4 \mathrm{~Hz}, \mathrm{H}_{6}\right), 4.95(\mathrm{t}, 1 \mathrm{H}, J=9.64 \mathrm{~Hz}$, $\left.\mathrm{H}_{4}\right), 5.1\left(\mathrm{dd}, 1 \mathrm{H}, J=1.8,5.04 \mathrm{~Hz}, \mathrm{H}_{1}\right), 5.37\left(\mathrm{ddd}, 1 \mathrm{H}, J=5.04,9.64,11.4 \mathrm{~Hz}, \mathrm{H}_{3}\right), 7.2-7.4(\mathrm{~m}, 5 \mathrm{H}, \mathrm{Ar}) .{ }^{13} \mathrm{C}$ NMR $\left(100 \mathrm{MHz}, \mathrm{CDCl}_{3}\right): \delta 20.70,20.75,20.9,35.3,62.3,64.4,69.2,69.9,71.1,84.4,88.5,121.7,128.3,128.9,131.9$, $169.8,170.2,170.7$.

\section{Compound 8}

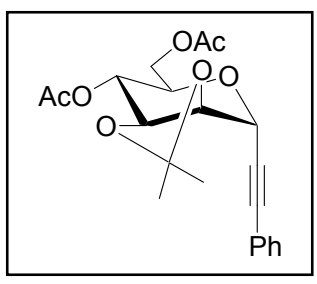

Obtained according to the typical procedure of alkynylation.

IR (ATR) : 1735, 1220, 758,724 $\mathrm{cm}^{-1}$.

HRMS-CI : $m / z[\mathrm{M}+\mathrm{Na}]^{+}$calcd for $\mathrm{C}_{21} \mathrm{H}_{24} \mathrm{O}_{7} \mathrm{Na}: 411.1427$, found : 411.1420 .

$[\alpha]_{\mathrm{D}}^{20}:-54\left(\mathrm{c}=0.94, \mathrm{CHCl}_{3}\right)$

${ }^{1} \mathrm{H}$ NMR $\left(400 \mathrm{MHz}, \mathrm{CDCl}_{3}\right): \delta 1.35\left(\mathrm{~s}, 3 \mathrm{H}, \mathrm{CH}_{3}\right), 1.5\left(\mathrm{~s}, 3 \mathrm{H}, \mathrm{CH}_{3}\right), 2.0(\mathrm{~m}, 6 \mathrm{H}, \mathrm{Ac}) ; 4.04(\mathrm{ddd}, 1 \mathrm{H}, J=2.28,5.48$, $10.32 \mathrm{~Hz}, \mathrm{H}-5$ ), 4.08 (dd, $1 \mathrm{H}, J=2.3,12.36 \mathrm{~Hz}, \mathrm{H}-6$ ), 4.22 (dd, $1 \mathrm{H}, J=5.48,12,36 \mathrm{~Hz}, \mathrm{H}-6$ '), 4.27 (dd, 1H, $J=5,48$, $9.8 \mathrm{~Hz}, \mathrm{H}-3), 4.34$ (dd, 1H, $J=1.4,5.5 \mathrm{~Hz}, \mathrm{H}-2), 5.06$ (dd, $1 \mathrm{H}, J=9.8,10.3 \mathrm{~Hz}, \mathrm{H}-4) ; 5.18$ (s, 1H, H-1), 7.3 (m, 5H, $\mathrm{Ar}) ;{ }^{13} \mathrm{C}$ NMR $\left(100 \mathrm{MHz}, \mathrm{CDCl}_{3}\right): \delta 20.8,20.9,26.5,27.6,62.5,66.3,69.5,69.9,75.8,77,83.3,88.4,110.5,121.5$, $128.4,129.1,131.8,169.7,170.8$.

\section{Compound 10}

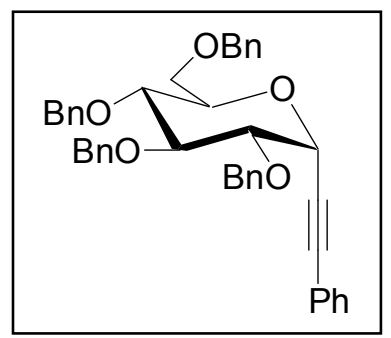

Obtained according to the typical procedure of alkynylation.

IR (ATR): 694, 734, $1070 \mathrm{~cm}^{-1}$.

HRMS-EI : $m / z[\mathrm{M}+\mathrm{H}]^{+}$calcd for $\mathrm{C}_{42} \mathrm{H}_{40} \mathrm{O}_{5} .: 624.2876$; found : 624.2867 .

$10 \alpha^{1} \mathrm{H}$ NMR (400MHz, $\left.\mathrm{CDCl}_{3}\right): \delta 3.68-3.84$ (m, 4H, H-2, H-3, H-6, H-6’), 4.06 (dd, 1H, J= 9.16, 9.6 Hz, H-4), 4.12 (ddd, $1 \mathrm{H}, J=1.84,3.2,9.6 \mathrm{~Hz}, \mathrm{H}-5), 4.54(\mathrm{~d}, 1 \mathrm{H}, \mathrm{Bn}), 4.55(\mathrm{~d}, 1 \mathrm{H}, \mathrm{Bn}), 4.65(\mathrm{~d}, 1 \mathrm{H}, \mathrm{Bn}), 4.88(\mathrm{~m}, 2 \mathrm{H}, \mathrm{Bn}), 5.05(\mathrm{~d}$, $1 \mathrm{H}, \mathrm{Bn}), 5.07(\mathrm{~d}, 1 \mathrm{H}, J=5.48 \mathrm{~Hz}, \mathrm{H}-1), 7.1-7.4(\mathrm{~m}, 25 \mathrm{H}, \mathrm{Ar}) .{ }^{13} \mathrm{C}$ NMR $\left(100 \mathrm{MHz}, \mathrm{CDCl}_{3}\right): \delta 67.3,68.7,72.8,73.6$, $73.8,75.4,75.8,77.7,79.4,83.3,84,122.5,128.2,128.5,132.0,138.2,138.9$.

$10 \beta{ }^{1} \mathrm{H}$ NMR $\left(400 \mathrm{MHz}, \mathrm{CDCl}_{3}\right): \delta 3.42(\mathrm{ddd}, 1 \mathrm{H}, J=2.28,4.6,11.9 \mathrm{~Hz}, \mathrm{H}-5), 3.55-3.7$ (m, 5H, H-2, H-3, H-4, H-6, H-6'), 4.22 (d, 1H, J = 9.16 Hz, H-1), 4.46 (d, 1H, Bn), 4.48 (d, 1H, Bn), 4.56 (d, 1H, Bn), 4.7-4.8 (m, 3H, Bn), 4.86 
(d, 1H, Bn), 5 (d, 1H, Bn), 7-7.4 (m, 25H, Ar). ${ }^{13} \mathrm{C}$ NMR $\left(100 \mathrm{MHz}, \mathrm{CDCl}_{3}\right): \delta 68.8,70.6,73.7,75.2,75.6,75.8,77.8$ $79.2,82.5,86.1,86.2,86.3,122.5,127.8,128.5,132.0,138.3,138.6$.

\section{Compound 12}

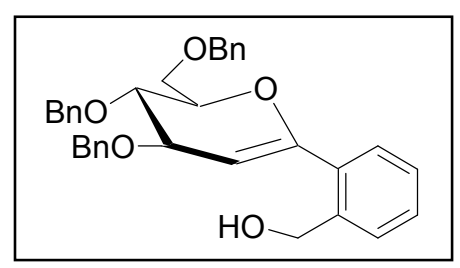

Obtained according to the typical procedure of alkynylation.

IR (ATR): 699, 748, $3600 \mathrm{~cm}^{-1}$.

HRMS-CI : $m / z[\mathrm{M}+\mathrm{Na}]^{+}$calcd for $\mathrm{C}_{34} \mathrm{H}_{34} \mathrm{O}_{5} \mathrm{Na}: 545.2328$ found : 545.2328

$[\alpha]_{\mathrm{D}}^{20}:-22\left(\mathrm{c}=0.6, \mathrm{CHCl}_{3}\right)$

${ }^{1} \mathrm{H}$ NMR $\left(250 \mathrm{MHz}, \mathrm{CDCl}_{3}\right): \delta 3.38(\mathrm{~m}, 2 \mathrm{H}, \mathrm{H}-3), 3.6$ (s, 1H, H-4), 3.6 (dd, $\left.1 \mathrm{H}, J=6.8,5.9 \mathrm{~Hz}, \mathrm{H}-6\right), 3.9(\mathrm{~d}, 1 \mathrm{H}, J=$ $7.3 \mathrm{~Hz}, \mathrm{H}-6$ '), 4.3 (d, 1H, $J=11.5, \mathrm{Bn}), 4.48$ (d, 1H, $J=11.5 \mathrm{~Hz}, \mathrm{Bn}), 4.57-4.7$ (m, 7H, H-5, Bn), 5.5 (s,1H, H-2), 7.2$7.3(\mathrm{~m}, 19 \mathrm{H}, \mathrm{Ar}) ;{ }^{13} \mathrm{C}$ NMR $\left(62.9 \mathrm{MHz}, \mathrm{CDCl}_{3}\right): \delta \quad 65.3,65.5,71.3,72,72.2,74.5,76.1,76.2,76.5,100.7,128.2$ $128.5,130.0,138.8$.

\section{Compound 14}

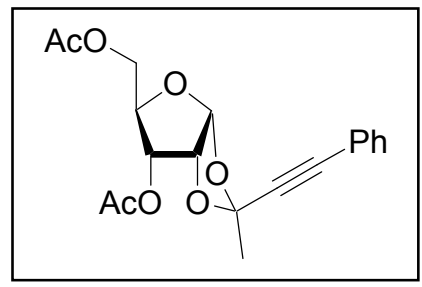

Obtained according to the typical procedure of alkynylation.

HRMS-CI : $m / z[\mathrm{M}+\mathrm{Na}]^{+}$calcd for $\mathrm{C}_{19} \mathrm{H}_{20} \mathrm{O}_{7} \mathrm{Na}: 383.1107$; found 383.1111 .

IR (ATR): 691, 758, 1222, 1741, $2231 \mathrm{~cm}^{-1}$.

14 major : ${ }^{1} \mathrm{H}$ NMR $\left(250 \mathrm{MHz}, \mathrm{CDCl}_{3}\right): \delta 1.8\left(\mathrm{~s}, 3 \mathrm{H}, \mathrm{CH}_{3}\right), 2.1(2 \mathrm{~s}, 6 \mathrm{H}, \mathrm{Ac}), 4.1$ (dd, $\left.1 \mathrm{H}, J=7,12.1 \mathrm{~Hz}, \mathrm{H}-5\right), 4.25$ (ddd, $1 \mathrm{H}, J=7,2.4,9.3 \mathrm{~Hz}, \mathrm{H}-4), 4.3$ (dd, $1 \mathrm{H}, J=2.4,12.1 \mathrm{~Hz}, \mathrm{H}-5), 4.7$ (dd, $1 \mathrm{H}, J=4.8,9.3 \mathrm{~Hz}, \mathrm{H}-3$ ), 4.9 (dd, $1 \mathrm{H}, J$ $=4.8,4 \mathrm{~Hz}, \mathrm{H}-2), 5.59(\mathrm{~d}, 1 \mathrm{H}, J=4 \mathrm{~Hz}, \mathrm{H}-1) .{ }^{13} \mathrm{C}$ NMR $\left(62.9 \mathrm{MHz}, \mathrm{CDCl}_{3}\right): \delta 19.7,25.5,61,71,74.4,76.3,82.8$, $85.4,102.6,103.5,120.4,127.3,128,130.8,169.0,169.6$.

14 minor : ${ }^{1} \mathrm{H}$ NMR $\left(250 \mathrm{MHz}, \mathrm{CDCl}_{3}\right): \delta 1.8\left(\mathrm{~s}, 3 \mathrm{H}, \mathrm{CH}_{3}\right), 2.1(2 \mathrm{~s}, 6 \mathrm{H}, \mathrm{Ac}), 5(\mathrm{dd}, 1 \mathrm{H}, J=4,4.6 \mathrm{~Hz}, \mathrm{H}-2), 5.95(\mathrm{~d}$, $1 \mathrm{H}, J=4 \mathrm{~Hz}, \mathrm{H}-1) .{ }^{13} \mathrm{C} \mathrm{NMR}\left(62.9 \mathrm{MHz}, \mathrm{CDCl}_{3}\right): \delta 19.7,25.5,81.5,85,100.7,104.0$. 


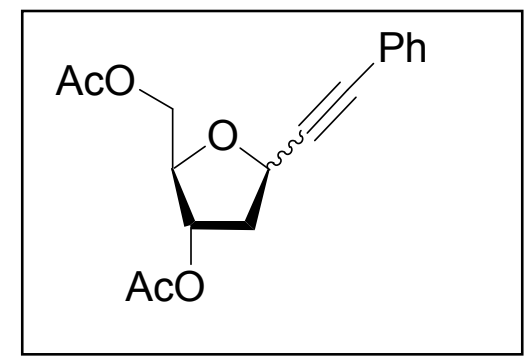

Obtained according to the typical procedure of alkynylation.

HRMS-CI : $m / z[\mathrm{M}+\mathrm{H}]^{+}$calcd for $\mathrm{C}_{17} \mathrm{H}_{19} \mathrm{O}_{5}: 303.1232$ found : 303.1231 .

IR (ATR): 691, 758, 1226, $1738 \mathrm{~cm}^{-1}$.

16 minor: ${ }^{1} \mathrm{H}$ NMR $\left(400 \mathrm{MHz}, \mathrm{CDCl}_{3}\right.$ ) $: \delta 2.1$ (s, 6H, Ac), 2.27 (ddd, $\left.1 \mathrm{H}, J=3.2,3.6,13.8 \mathrm{~Hz}, \mathrm{H}-2\right), 2.63$ (ddd, $1 \mathrm{H}, J$ $=7.3,7.3,13.8 \mathrm{~Hz}, \mathrm{H}-2), 4.2(\mathrm{dd}, 1 \mathrm{H}, J=5.5,11.9 \mathrm{~Hz}, \mathrm{H}-5), 4.3(\mathrm{dd}, 1 \mathrm{H}, J=3.6,3.6,11.9 \mathrm{~Hz}, \mathrm{H}-5), 4.39$ (ddd, $1 \mathrm{H}, J$ $=3.6,5.5 \mathrm{~Hz}, \mathrm{H}-4), 5.08$ (dd, $1 \mathrm{H}, J=3.2,7.3 \mathrm{~Hz}, \mathrm{H}-1), 5.13$ (ddd, $1 \mathrm{H}, J=3.2,3.6,7.3 \mathrm{~Hz}, \mathrm{H}-3), 7.3-7.5$ (m, $5 \mathrm{H}, \mathrm{Ar}$ ). ${ }^{13} \mathrm{C}$ NMR $\left(100 \mathrm{MHz}, \mathrm{CDCl}_{3}\right): \delta 20.6,20.7,39.2,63.6,68.2,74.9,81,8.4,87.5,122.2,128.0,128.4,131.6,170.0$.

16 minor: ${ }^{1} \mathrm{H}$ NMR $\left(400 \mathrm{MHz}, \mathrm{CDCl}_{3}\right): \delta 2.1(\mathrm{~s}, 6 \mathrm{H}, \mathrm{Ac}), 4.18$ (m, $\left.1 \mathrm{H}, \mathrm{H}-4\right), 4.35$ (dd, $\left.1 \mathrm{H}, J=4.1,11.4 \mathrm{~Hz}, \mathrm{H}-5\right), 5.44$ (ddd, $1 \mathrm{H}, J=3.68,3.68,9.6 \mathrm{~Hz}, \mathrm{H}-1), 7.2-7.5$ (m, 5H, Ar). ${ }^{13} \mathrm{C}$ NMR $\left(100 \mathrm{MHz}, \mathrm{CDCl}_{3}\right): \delta 20.6,20.7,39.4,63.9$, $68.4,75.6,82.1,85.2,86.5,122.2,128.0,128.4,131.6,170.1$.

\section{Compound 18}

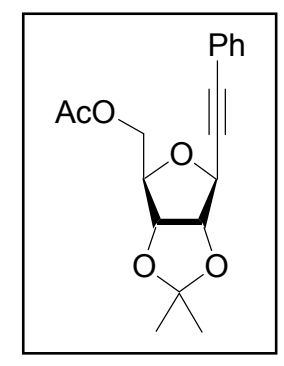

Obtained according to the typical procedure of alkynylation.

HRMS-CI : $m / z[\mathrm{M}+\mathrm{Na}]^{+}$calcd for $\mathrm{C}_{18} \mathrm{H}_{20} \mathrm{O}_{5} \mathrm{Na}: 339.1208$ found : 339.1215 .

IR (ATR) : 691, 757, 1233, 1742, $2230 \mathrm{~cm}^{-1}$.

18ß: ${ }^{1} \mathrm{H}$ NMR $\left(400 \mathrm{MHz}, \mathrm{CDCl}_{3}\right): \delta 1.44\left(\mathrm{~s}, 3 \mathrm{H}, \mathrm{CH}_{3}\right), 1.65\left(\mathrm{~s}, 3 \mathrm{H}, \mathrm{CH}_{3}\right), 2.1(\mathrm{~s}, 3 \mathrm{H}, \mathrm{Ac}), 4.26(\mathrm{dd}, 1 \mathrm{H}, J=4.6,9.6$ $\mathrm{Hz}, \mathrm{H}-5$ ), 4.32 (ddd, $1 \mathrm{H}, J=4.6,5.5,7.8 \mathrm{~Hz}, \mathrm{H}-4), 4.37$ (dd, 1H, $J=5.5,9.6 \mathrm{~Hz}, \mathrm{H}-5$ ), 4.74 (dd, $1 \mathrm{H}, J=2.28,5.96 \mathrm{~Hz}$, $\mathrm{H}-3), 4.91(\mathrm{dd}, 1 \mathrm{H}, J=2.44,5.5 \mathrm{~Hz}, \mathrm{H}-2), 4.93(\mathrm{~d}, 1 \mathrm{H}, J=2.44 \mathrm{~Hz}, \mathrm{H}-1), 7.2-7.4(\mathrm{~m}, 5 \mathrm{H}, \mathrm{Ar}) .{ }^{13} \mathrm{C} \mathrm{NMR}(100 \mathrm{MHz}$, $\left.\mathrm{CDCl}_{3}\right): \delta 21.0,25.4,26.9,64.0,45.3,82.9,83.6,86.2,86.5,87.0,114.0,122.1,128.4,128.8,131.7,170.8$.

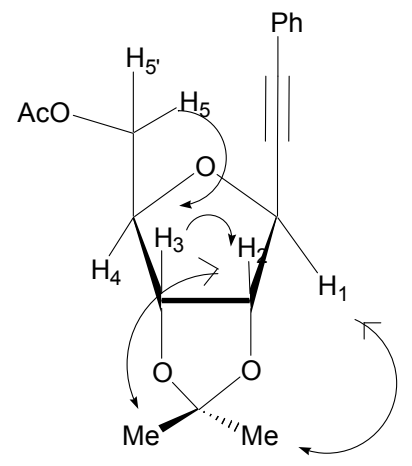

2D NOESY Correlations 


\section{Compound 20}

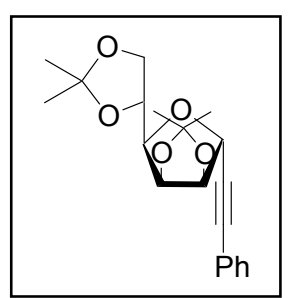

Obtained according to the typical procedure of alkynylation.

HRMS-CI : $m / z[\mathrm{M}+\mathrm{H}]^{+}$calcd for $\mathrm{C}_{20} \mathrm{H}_{25} \mathrm{O}_{5}: 345.1702$; found : 345.1700 .

IR (ATR): 691, $759 \mathrm{~cm}^{-1}$.

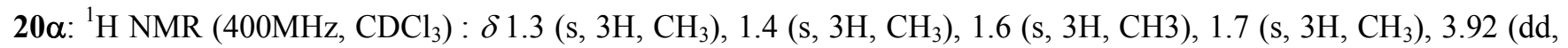
$1 \mathrm{H}, J=3.2,7.8 \mathrm{~Hz}, \mathrm{H}-4), 4.02$ (m, 2H, H-6, H-6'), 4.35 (ddd, $1 \mathrm{H}, J=5.5,5.5,7.8 \mathrm{~Hz}, \mathrm{H}-5$ ), 4.78 (dd, $1 \mathrm{H}, J=3.2$, $5.96 \mathrm{~Hz}, \mathrm{H}-3), 4.81(\mathrm{~d}, 1 \mathrm{H}, J=5.96 \mathrm{~Hz}, \mathrm{H}-2), 4.86(\mathrm{~s}, 1 \mathrm{H}, \mathrm{H}-1), 7.2-7.4(\mathrm{~m} 5 \mathrm{H}, \mathrm{Ar}) .{ }^{13} \mathrm{C} \mathrm{NMR}\left(100 \mathrm{MHz}, \mathrm{CDCl}_{3}\right): \delta$ 24.7, 25.3, 26.0, 27.1, 67.1, 73.1, 74.5, 80.5, 81.4, 84.5, 86.2, 87.1, 109.1, 112.7, 121.8, 128.2, 128.6, 131.6.

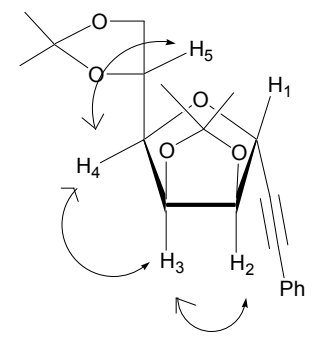

\section{D NOESY Correlations}

20ß : ${ }^{1} \mathrm{H}$ NMR $\left(400 \mathrm{MHz}, \mathrm{CDCl}_{3}\right): \delta 1.35\left(\mathrm{~s}, 3 \mathrm{H}, \mathrm{CH}_{3}\right), 1.53\left(\mathrm{~s}, 3 \mathrm{H}, \mathrm{CH}_{3}\right), 1.57\left(\mathrm{~s}, 3 \mathrm{H}, \mathrm{CH}_{3}\right), 1.7\left(\mathrm{~s}, 3 \mathrm{H}, \mathrm{CH}_{3}\right), 3.77$ (dd, 1H, $J=3.2,11 \mathrm{~Hz}, \mathrm{H}-4), 4-4.1$ (m, H-6, H-6'), 4.27 (m, H-5).

\section{Compound 22}

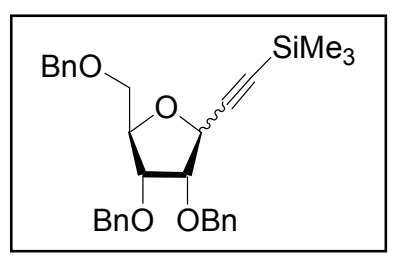

Obtained according to the typical procedure of alkynylation.

HRMS-EI : $m / z[\mathrm{M}+]^{+}$calcd for $\mathrm{C}_{31} \mathrm{H}_{36} \mathrm{O}_{4} \mathrm{Si} ; 500.2383$ found 500.2368

IR (ATR) 696, 750, 1060, $2188 \mathrm{~cm}^{-1}$. 
22: ${ }^{1} \mathrm{H}$ NMR (400MHz, $\mathrm{CDCl}_{3}$ ) : $\delta: 0,16$ (s, 9H, $\mathrm{SiMe}_{3}$ ) ; 3,53-3,62 (m, 2H, H-5), 3,97-4,3 (m, 2H, H-2, H-3), 4,124,19 (m, 1H, H-4), 4,65 (s, 1H, H-1), 4,45-4,75 (m, 6H, H-6, H-7, H-8), 7,2 (m, 15H, H-12, H-13, H-14). ${ }^{13} \mathrm{C}$ NMR $\left(100 \mathrm{MHz}, \mathrm{CDCl}_{3}\right): \delta: 0,70.3,71.6,72.4,73.6,72.5,78.6,81.7,82.2,92.0,103.3,127.2,128.5,129.0,137.9,138.0$, 138.4 .

22': ${ }^{1} \mathrm{H}$ NMR (400MHz, $\left.\mathrm{CDCl}_{3}\right): \delta: 0,15\left(\mathrm{~s}, 9 \mathrm{H}, \mathrm{SiMe}_{3}\right), 3,53-3,6(\mathrm{dd}, 1 \mathrm{H}, \mathrm{H}-5, J=3.66,11 \mathrm{~Hz}, \mathrm{H}-5), 3,68-3,74$ (dd, $1 \mathrm{H}, J=11,2.76 \mathrm{~Hz}, \mathrm{H}-5), 4,05$ (m, 2H, $J=3.24,4.12 \mathrm{~Hz}, \mathrm{H}-2, \mathrm{H}-3), 4,28-4,34$ (m, 1H, $J=3.66,2.76,4.12 \mathrm{~Hz}, \mathrm{H}-4)$, 4,74-4,77 (m, $1 \mathrm{H}, J=4.12 \mathrm{~Hz}, \mathrm{H}-1), 4,38-5,12(6 \mathrm{H}, \mathrm{m}, \mathrm{H}-6, \mathrm{H}-7, \mathrm{H}-8), 7,2-7,5$ (m, $15 \mathrm{H}, \mathrm{Ar}) .{ }^{13} \mathrm{C}$ NMR $(100 \mathrm{MHz}$, $\left.\mathrm{CDCl}_{3}\right): \delta: 0,69.5,71.4,72.4,73.1,73.6,78.0,78,6,80.0,93.2 ; 103.2 ; 127.2,128.5,129.0 ; 138.0,138.3,138.4$.

\section{Compound 24}

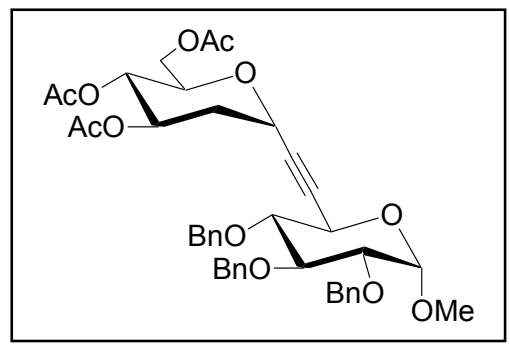

Obtained according to the typical procedure of alkynylation.

HRMS-CI : $m / z\left[\mathrm{M}+\mathrm{NH}_{4}\right]^{+}$calcd for $\mathrm{C}_{41} \mathrm{H}_{50} \mathrm{NO}_{12}$ 748.3333, found 748.3328.

IR (ATR): 698,753, 1748, $2233 \mathrm{~cm}^{-1}$.

$[\alpha]_{\mathrm{D}}^{20}:-5\left(\mathrm{c}=1.2, \mathrm{CHCl}_{3}\right)$

${ }^{1} \mathrm{H}$ NMR (400MHz, $\left.\mathrm{CDCl}_{3}\right): \delta$ 1.87-2.12 (m, 9H, Ac), $2.2(\mathrm{~m}, 2 \mathrm{H}, \mathrm{H}-9), 3.3$ (s, 3H, OMe), 3.45 (dd, 1H, $J=11.92$, 1.84, H-2), 3.49 (t, 1H, H-4), 3.8-3.9 (m, 2H, H-13 and H-3), 3.94-4.1 (m, 1H, H-12), 4.18 (dd, $1 \mathrm{H}, J=12.36,4.12$ $\mathrm{Hz}, \mathrm{H}-13), 4.38$ (d, $1 \mathrm{H}, J=11 \mathrm{~Hz}, \mathrm{H}-5), 4.65(\mathrm{~d}, 1 \mathrm{H}, J=11.92, \mathrm{H}-1), 4.7-4.9\left(\mathrm{~m}, 8 \mathrm{H}, \mathrm{H}-8\right.$ and H-11, $\left.\mathrm{CH}_{2} \mathrm{Bn}\right), 5.28$ (m, 1H, H-10), 7.3 (m, 15H, Ar). ${ }^{13} \mathrm{C}$ NMR $\left(100 \mathrm{MHz}, \mathrm{CDCl}_{3}\right): \delta$ 20.6-20.8, 35.1, 55.9, 62.2, 64.1, 72, 71.4, 73.5, 76.2, $78.8,80.9,81.0,82.1,85.8,98.3,127.8,128.2,128.5,138.0,138.3,138.6,169.6,169.9,170.6$.

\section{Compound 25}

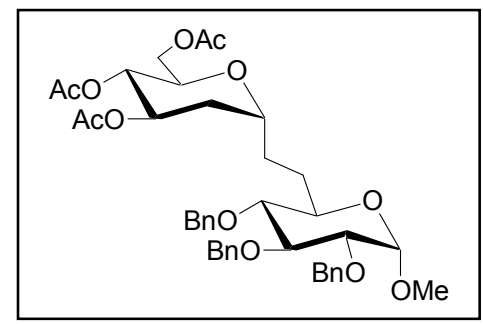

HRMS-CI : $m / z[\mathrm{M}+\mathrm{Na}]^{+}$calcd for $\mathrm{C}_{26} \mathrm{H}_{38} \mathrm{O}_{15} \mathrm{Na}: 613.2108$, found : 613.2106.

IR (ATR): $2960,1740,1451,1368,1217,1035 \mathrm{~cm}^{-1}$

$[\alpha]_{\mathrm{D}}^{20}:+79\left(\mathrm{c} 1, \mathrm{CHCl}_{3}\right)$ 
${ }^{1} \mathrm{H}$ NMR $\left(400 \mathrm{MHz}, \mathrm{CDCl}_{3}\right): \delta 1.54$ (m, 4H, H-6, H-7), 1.83 (ddd, $1 \mathrm{H}, J=13.2,4.6,8.72 \mathrm{~Hz}, \mathrm{H}-9$ ), 1.93 (ddd, $1 \mathrm{H}, J=$ $14.2,6,4.56 \mathrm{~Hz}, \mathrm{H}-9), 3.32$ (s, $3 \mathrm{H}, \mathrm{OMe}$ ), 3.75 (q, $1 \mathrm{H}, J=10.56,5.92 \mathrm{~Hz}, \mathrm{H}-5), 3.85$ (dt, 1H, $J=6.88,3.82 \mathrm{~Hz}, \mathrm{H}-$ 12), 3.95 (m, 1H, H-8), 3.97 (dd, $1 \mathrm{H}, J=3.2,12.36 \mathrm{~Hz}, \mathrm{H}-13), 4.42$ (dd, $1 \mathrm{H}, J=6.44 \mathrm{~Hz}, \mathrm{H}-13$ ), 4.84 (m, 3H, H-2, H4, H-11), 4.88 (d, $1 \mathrm{H}, J=3.68 \mathrm{~Hz}, \mathrm{H}-1), 5.1$ (dt, 1H, $J=4.56,8.24 \mathrm{~Hz}, \mathrm{H}-10), 5.43$ (t, 1H, $J=10 \mathrm{~Hz}, \mathrm{H}-3), 7.2-7.4(\mathrm{~m}$, $5 \mathrm{H}, \mathrm{Ar}) .{ }^{13} \mathrm{C} \mathrm{NMR}\left(100 \mathrm{MHz}, \mathrm{CDCl}_{3}\right): \delta 21.0,27.2,33.0,55.5,62.0,67.8,68.2,69.9,70.3,71.2,72.3,96.6,169.9$, $170.2,170.3$. 


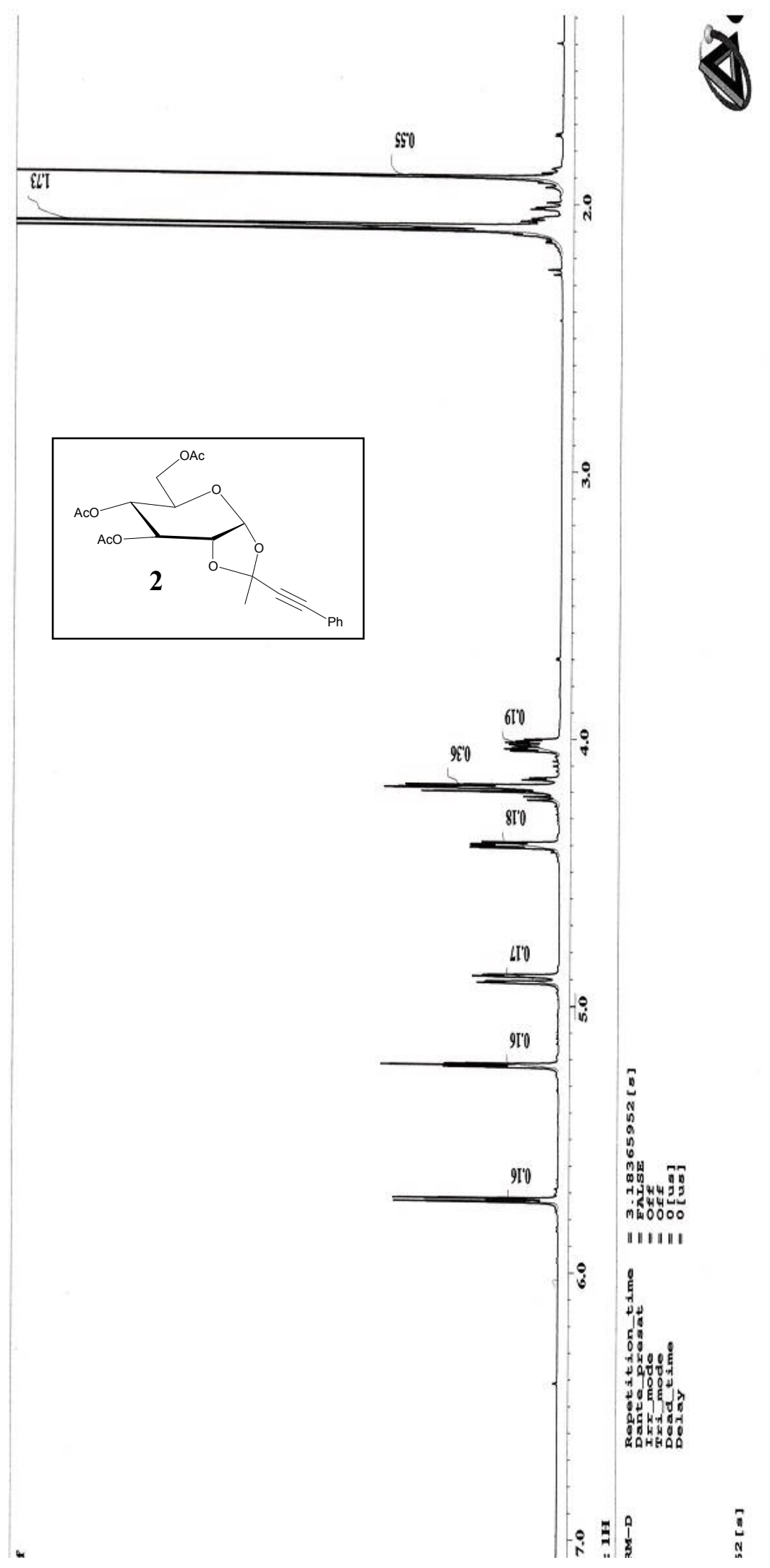

S-12 


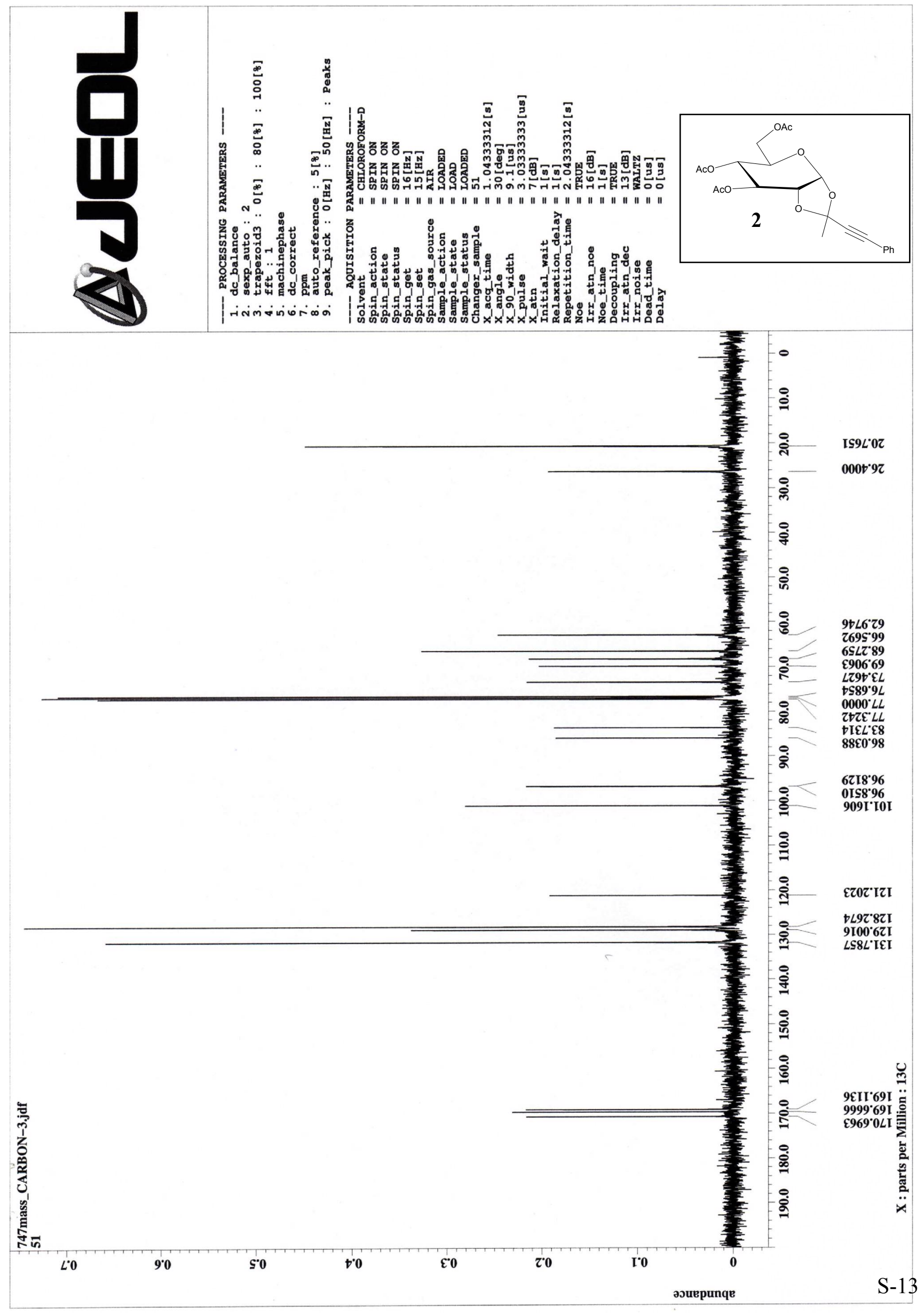



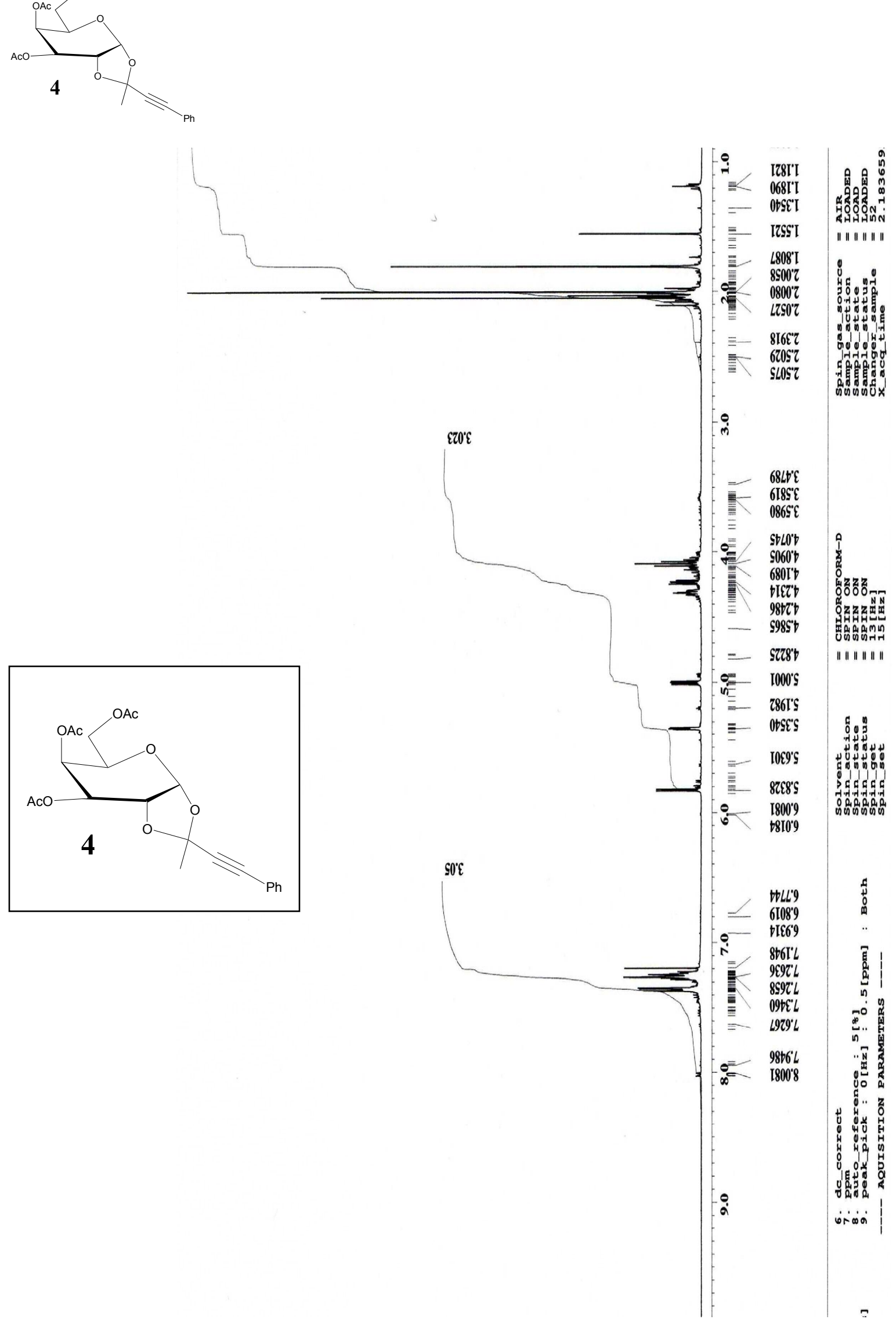


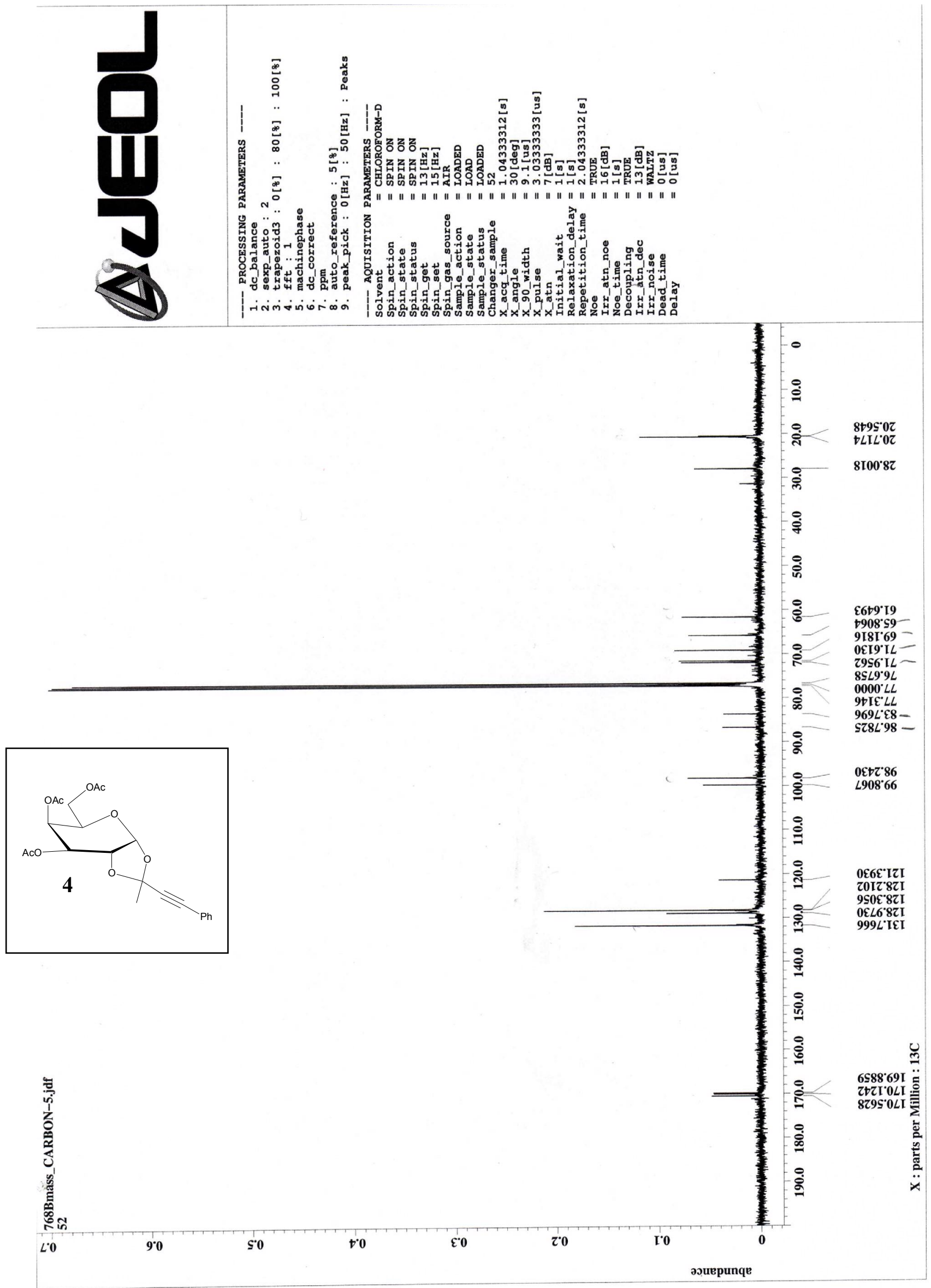




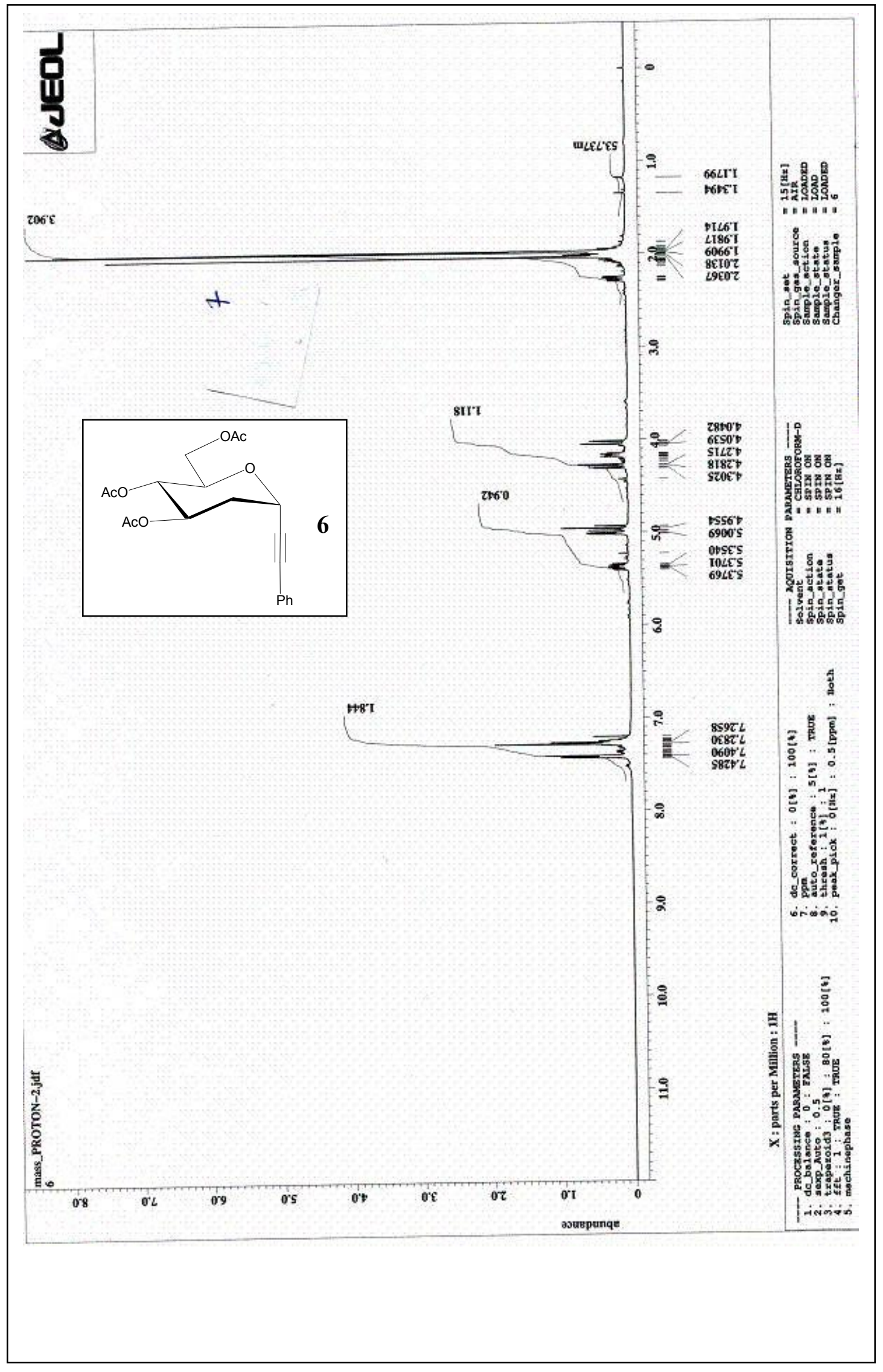




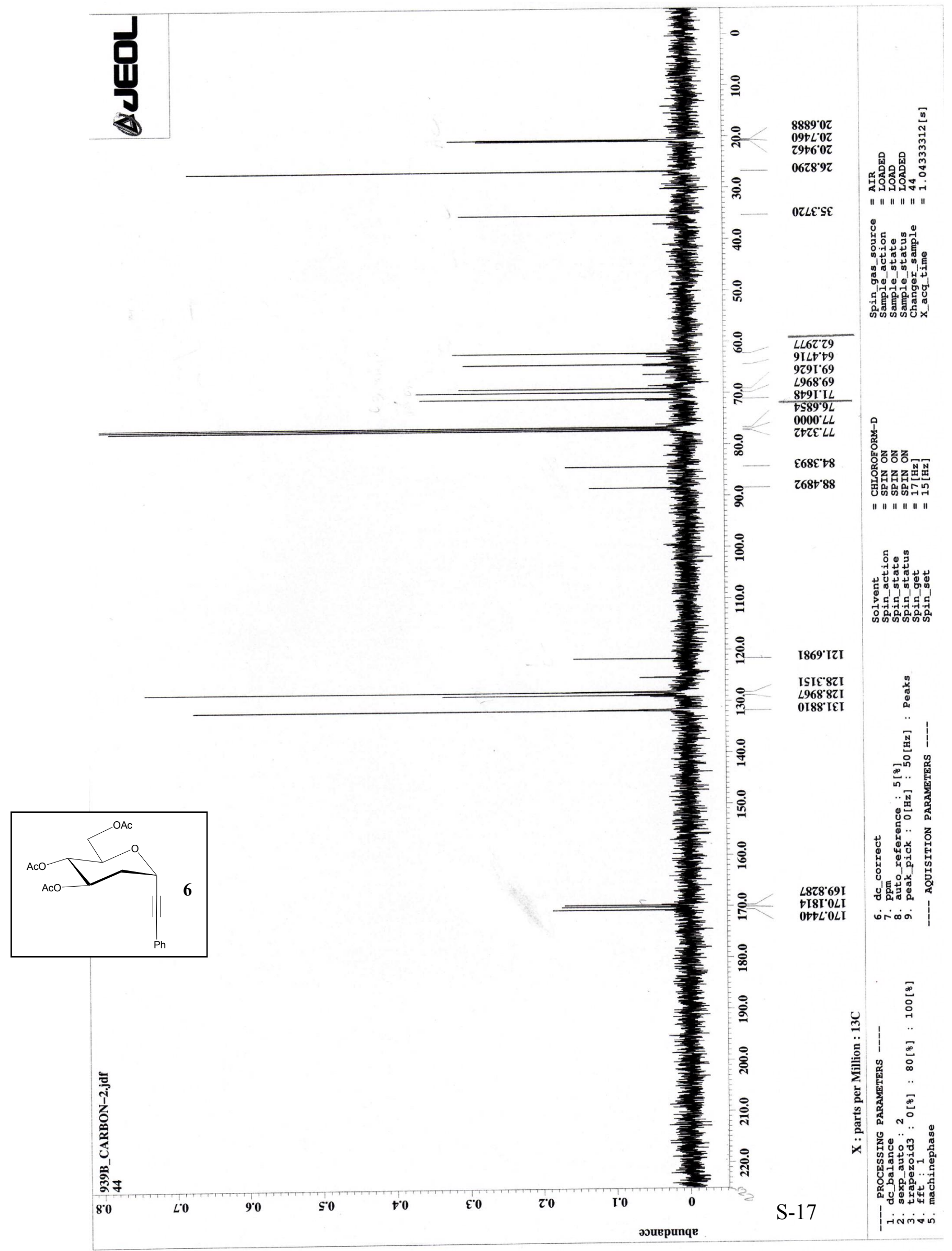




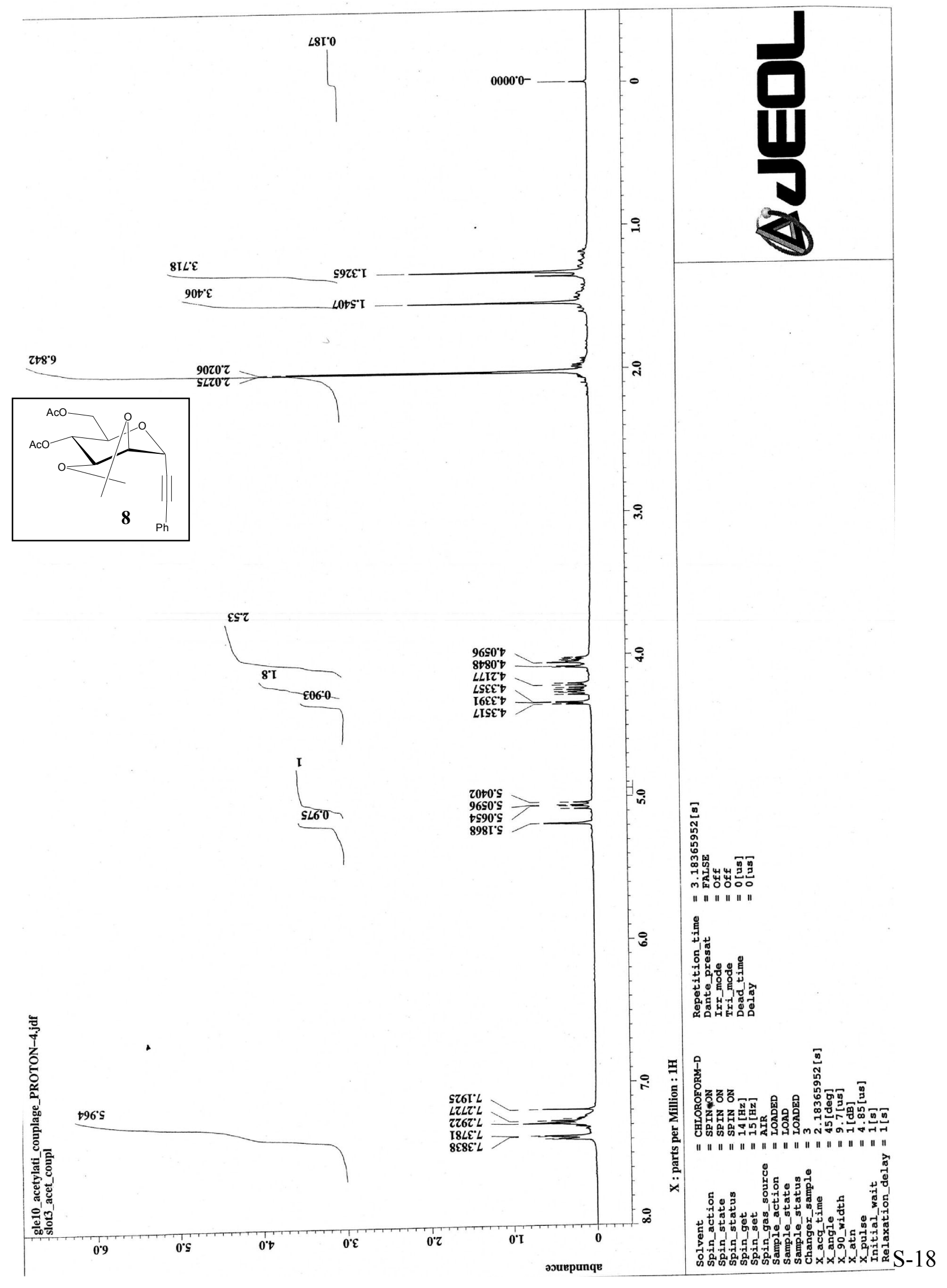




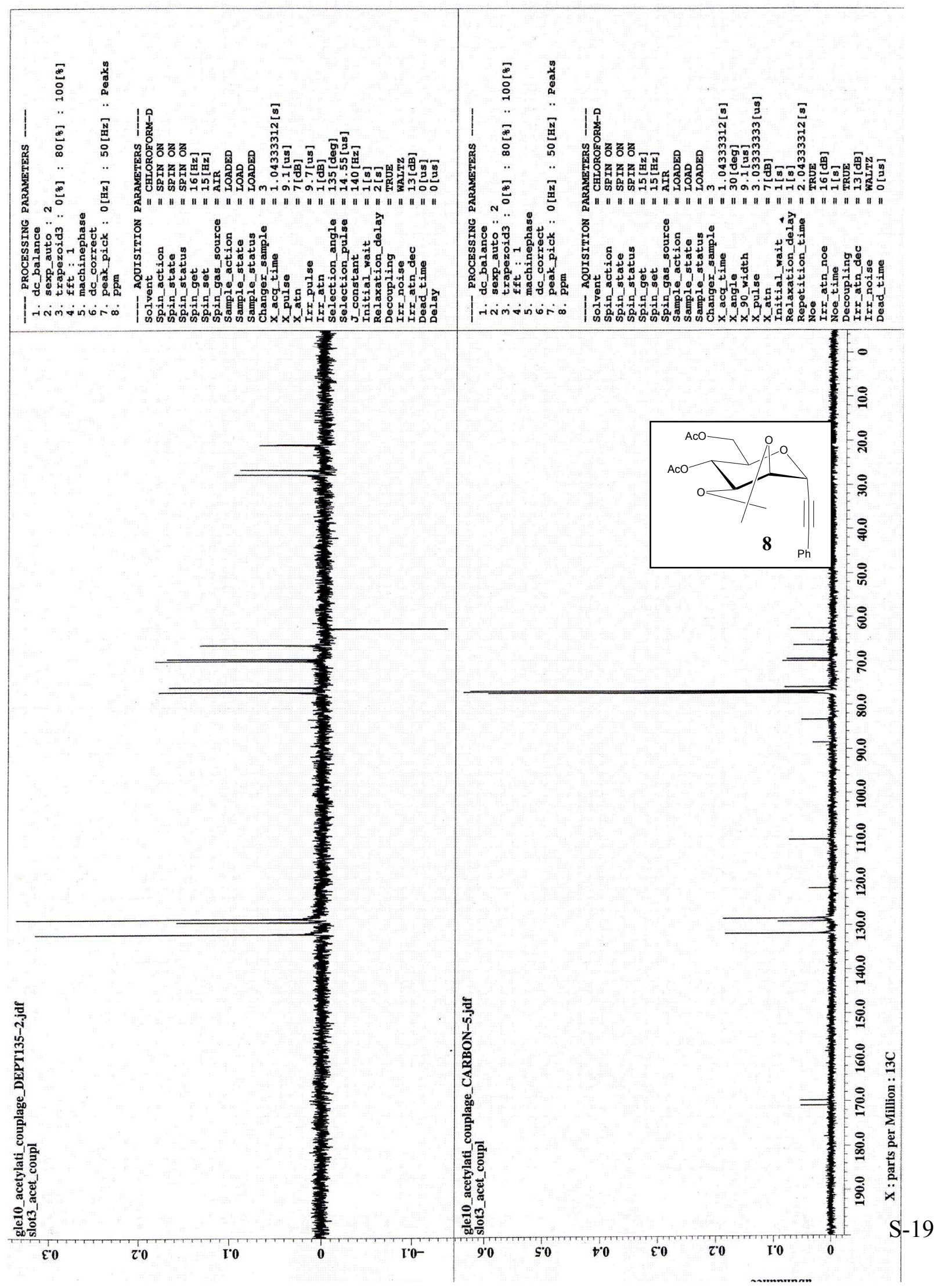




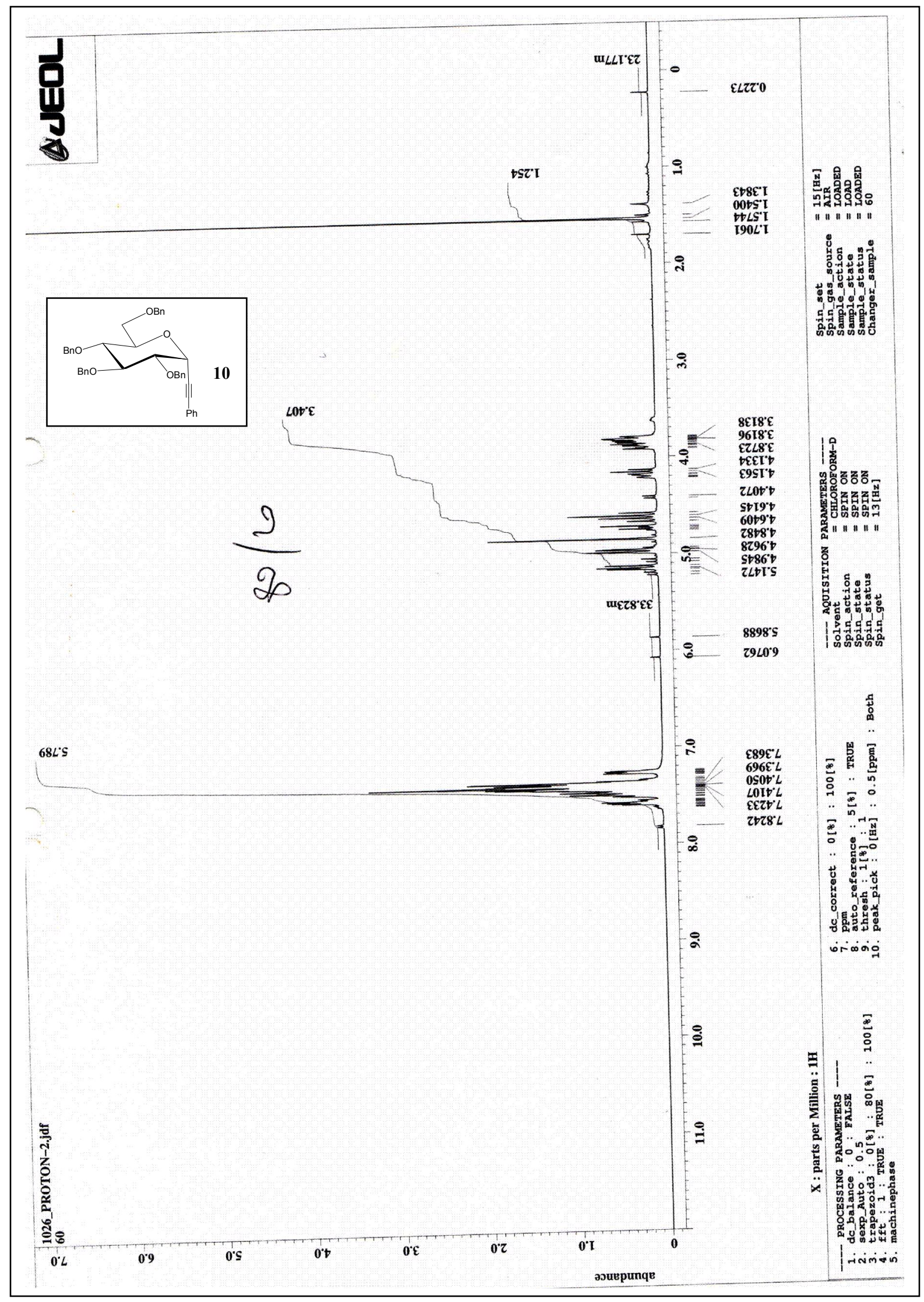

